\title{
A test of HHCPT using magnetic moments of heavy baryons
}

\section{Scimemi}

Dep. de Fúsica Teórica, IFIC, Univ. de Valencia-CSIC,

Edificio de Institutos de Investigación de Paterna

Aptdo. de Correos 2085 E-46071 Valencia, Spain *

ABSTRACT: First non-trivial chiral corrections to the magnetic moments of triplet $(T)$ and $\operatorname{sextet}\left(S^{(*)}\right)$ heavy baryons are presented using Heavy Hadron Chiral Perturbation Theory. The contributions are calculated up to order $\mathcal{O}\left(1 /\left(m_{Q} \Lambda_{\chi}^{2}\right)\right)$ for $T$-baryons and up to order $\mathcal{O}\left(1 / \Lambda_{\chi}^{2}\right)$ for $S^{(*)}$-baryons. The renormalization of the chiral loops is discussed and relations among the magnetic moments of different hadrons are provided.

\section{Introduction}

In some kinematical regions, which are not far from the chiral and heavy quark limits, both Chiral Perturbation Theory (ChPT) and Heavy Quark Effective Theory (HQET) can be combined together to construct an effective Lagrangian which describes soft interactions of hadrons

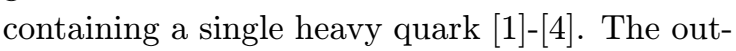
coming theory takes the name of Heavy Hadron Chiral Perturbation Theory (HHCPT). The basic fields of HHCPT are heavy hadrons and light mesons. In ref. [6], the formalism is extended to include also electromagnetism. We use this hybrid effective Lagrangian to calculate the magnetic moments (MM) of some baryons containing a $c$ or a $b$ quark. We find that within HHCPT, it is possible to establish relations among the MM of all this baryons. This measurement can provide a useful test for HHCPT. In this paper we review the issue as it has been presented in ref.

The light degrees of freedom in the ground state of a baryon with one heavy quark can be either in a $s_{l}=0$ or in a $s_{l}=1$ configuration. The first one corresponds to $J^{P}=\frac{1}{2}^{+}$baryons and are annihilated by $T_{i}(v)$ fields which trans-

\footnotetext{
${ }^{*}$ I thank A. Della Riccia Foundation (Florence, Italy) for support. I acnkowledge M.C. Bañuls, J. Bernabéu, V. Giménez, A.Pich for collaboration. Preprint FTUV/9976, IFIC/99-79
}

form as a $\overline{\mathbf{3}}$ under the chiral $S U(3)_{L+R}$ and as a doublet under the HQET $S U(2)_{v}$. In the second case, $s_{l}=1$, the spin of the heavy quark and the light degrees of freedom combine together to form $J^{P}=3 / 2^{+}$and $J=1 / 2^{+}$baryons which are degenerate in mass in the $m_{Q} \rightarrow \infty$ limit. The spin- $\frac{3}{2}$ ones are annihilated by the RaritaSchwinger field $S_{\mu}^{* i j}(v)$ while the spin- $\frac{1}{2}$ baryons are destroyed by the Dirac field $S^{i j}(v)$.

The particle assignement for the $J=1 / 2$ charmed baryons of the $\overline{\mathbf{3}}$ and $\mathbf{6}$ representations is $\left(T_{1}, T_{2}, T_{3}\right)=\left(\Xi_{c}^{0},-\Xi_{c}^{+}, \Lambda_{c}^{+}\right)$,

$$
S^{i j}=\left(\begin{array}{ccc}
\Sigma_{c}^{++} & \sqrt{\frac{1}{2}} \Sigma_{c}^{+} & \sqrt{\frac{1}{2}} \Xi_{c}^{+^{\prime}} \\
\sqrt{\frac{1}{2}} \Sigma_{c}^{+} & \Sigma_{c}^{0} & \sqrt{\frac{1}{2}} \Xi_{c}^{0^{\prime}} \\
\sqrt{\frac{1}{2}} \Xi_{c}^{+^{\prime}} & \sqrt{\frac{1}{2}} \Xi_{c}^{0^{\prime}} & \Omega_{c}^{0}
\end{array}\right),
$$

and the corresponding $b$-baryons are $\left(T_{1}, T_{2}, T_{3}\right)=\left(\Xi_{b}^{-},-\Xi_{b}^{0}, \Lambda_{b}^{0}\right)$,

$$
S^{i j}=\left(\begin{array}{ccc}
\Sigma_{b}^{+} & \sqrt{\frac{1}{2}} \Sigma_{b}^{0} & \sqrt{\frac{1}{2}} \Xi_{b}^{0^{\prime}} \\
\sqrt{\frac{1}{2}} \Sigma_{b}^{0} & \Sigma_{b}^{-} & \sqrt{\frac{1}{2}} \Xi_{b}^{-^{\prime}} \\
\sqrt{\frac{1}{2}} \Xi_{b}^{0^{\prime}} & \sqrt{\frac{1}{2}} \Xi_{b}^{-^{\prime}} & \Omega_{b}^{-}
\end{array}\right) .
$$

The $J=3 / 2$ partners of these $S$ baryons have the same $S U(3)_{V}$ assignement in $S_{\mu}^{* i j}$.

The chiral Lagrangian describing the soft hadronic and electromagnetic interactions of these baryons in the infinite heavy quark mass limit 
can be found in ref. [i-i] and we refer to this paper for its complete expression and notation. Here we point out only some considerations.

First of all, there are no MM terms in the lowest order Lagrangian. Therefore, the contributions to the MM come from:

1) the order $\mathcal{O}\left(1 / \Lambda_{\chi}\right)$ terms in the baryon chiral Lagrangian $\overline{i_{1}}, \overline{b_{1}}$. In the rest of the paper we will take $\Lambda_{\chi}=4 \pi f_{\pi} \simeq 1.2 \mathrm{GeV}$ which fixes the normalization of the unknown couplings $c_{i}$;

2 ) terms of order $1 / m_{Q}$ from the heavy quark expansion which break both spin and flavour sym-

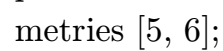

3) chiral loops of Goldstone bosons coupled to photons, as described by the lowest order Lagrangian;

4) we have not considered contributions of order $\mathcal{O}\left(1 /\left(m_{Q} \Lambda_{\chi}\right)\right)$. For the b-baryons, these corrections can be safely neglected. For the c-baryons, however, a simple estimate shows that their contribution cannot be larger than, say, $15 \%$. Moreover also $O\left(1 / \Lambda_{\chi}^{3}\right)$ contributions can be neglected because they are NNLO chiral corrections.

The renormalization of the divergent chiral loops which contribute to the MM requires the introduction of higher order operators. In the case of $S$ baryons we find that all divergences and scale dependence to $\mathcal{O}\left(1 / \Lambda_{\chi}^{2}\right)$ can be absorbed in a redefinition of only one $\mathcal{O}\left(1 / \Lambda_{\chi}\right)$ coupling. Results are presented in section $\overline{2}_{1}^{1}$ The magnetic moments of the $T$-baryons are analyzed in ref. [14]. However this analysis does not include all meson loops and the needed counterterms are not taken into account. In section a consistent calculation of the $T$ magnetic moments to order $\mathcal{O}\left(1 /\left(m_{Q} \Lambda_{\chi}^{2}\right)\right)$. Finally section summarizes our conclusions.

\section{Results for $S$-baryons $\left(s_{l}=1\right)$}

We define the magnetic moment operator for a spin $1 / 2$ baryon $B$ and a spin $3 / 2$ baryon $B_{\nu}^{*}$ respectively as

$$
-i e \mu(B) F^{\alpha \beta} \bar{B} \sigma_{\alpha \beta} B ;-i e \mu\left(B^{*}\right) F^{\alpha \beta} \bar{B}_{\mu}^{*} \sigma_{\alpha \beta} B^{* \mu} .
$$

The leading contributions from the light- and heavy-quark magnetic interactions are of order $\mathcal{O}\left(1 / \Lambda_{\chi}\right)$ and $\mathcal{O}\left(1 / m_{Q}\right)$ respectively. We com- pute the next-to-leading chiral corrections of or$\operatorname{der} \mathcal{O}\left(1 / \Lambda_{\chi}^{2}\right)$ which originate from the loop diagrams shown in fig. $\overline{1}_{1}^{\prime}$.

The resulting MM can be decomposed as:

$$
\begin{aligned}
& \mu\left(B^{(*)}\right)=\frac{1}{72}\left(6 \frac{Q_{Q}}{m_{Q}} \mu_{H Q E}\left(B^{(*)}\right)+\right. \\
& \frac{16 c_{s}}{\Lambda_{\chi}} \mu_{\chi}\left(B^{(*)}\right)+3 g_{2}^{2} \frac{\Delta_{S T}}{\left(4 \pi f_{\pi}\right)^{2}} \mu_{g_{2}}\left(B^{(*)}\right)- \\
& \left.3 g_{3}^{2} \frac{m_{K}}{4 \pi f_{\pi}^{2}} \mu_{g_{3}}\left(B^{(*)}\right)\right) .
\end{aligned}
$$

where $\mu_{i}(B)$ and $\mu_{i}\left(B^{*}\right)$ are related by

$$
\begin{aligned}
\frac{1}{3} \mu_{H Q E}\left(B^{*}\right) & =\mu_{H Q E}(B)=1 \\
\mu_{i}\left(B^{*}\right) & =-\frac{3}{2} \mu_{i}(B) \quad i=\chi, g_{2}, g_{3} .
\end{aligned}
$$

The values of the $\mu_{i}(B)$ contributions are reported in table $]_{1}^{\overline{1}}$ for baryons containing a $Q$ quark $(Q=c, b)$ where

$$
\begin{aligned}
I_{i} \equiv & I\left(\Delta_{S T}, m_{i}\right)=2\left(-2+\log \frac{m_{i}^{2}}{\mu^{2}}\right)+ \\
& 2 \frac{\sqrt{\Delta_{S T}^{2}-m_{i}^{2}}}{\Delta_{S T}} \log \left(\frac{\Delta_{S T}+\sqrt{\Delta_{S T}^{2}-m_{i}^{2}}}{\Delta_{S T}-\sqrt{\Delta_{S T}^{2}-m_{i}^{2}}}\right) .
\end{aligned}
$$

We want to stress that due to flavour symmetry, the constants $c_{s}, g_{2}$ and $g_{3}$, and hence the values of $\mu_{\chi}, \mu_{g_{2}}$ and $\mu_{g_{3}}$, are the same for $c$ and $b$-baryons. The only difference is the contribution proportional to $\mu_{H Q E}$ due to the different electric charge of the $c, Q_{c}=+2 / 3$, and $b$, $Q_{b}=-1 / 3$, quarks (see equation 2.27 ).

The results proportional to $g_{2}^{2}$ are obtained performing a one-loop integral (fig. $S$-baryon running in the loop) that has to be renormalized. The divergent part of the integral does not depend on the pion or kaon mass and is instead proportional to the mass of the baryon running in the loop. If one considers both pion and kaon loops the divergent part respects the $S U(3)$ structure of the chiral multiplet and can be canceled with an operator of the form

$$
\frac{e}{\Lambda_{\chi}^{2}} \operatorname{tr}\left[\bar{S}_{\mu}\left(v \cdot D S_{\nu}\right) Q-\left(v \cdot D \bar{S}_{\mu}\right) S_{\nu} Q\right] F^{\mu \nu} .
$$

This is the most general dimension- 6 chiral and Lorentz invariant operator constructed out of $S_{\mu}^{i j}$ 
and $Q F_{\mu \nu}$, preserving parity and time-reversal invariance which contributes to MM. When the equation of motion $\left((v \cdot D) S_{\mu}=\Delta_{S T} S_{\mu}\right)$ is applied, its contribution is of the same form as the term proportional to $c_{s}$. Thus, the local contribution from the operator in equation 2.4 can be taken into account, through an effective coupling $c_{S}(\mu)$. The scale $\mu$ dependence of the loop integrals is exactly canceled by the corresponding dependence of the coefficient $c_{S}(\mu)$.

The contribution proportional to $g_{3}^{2}$ involves a loop integral in which a baryon of the $T$-multiplet is running in the loop. However, as we are in the limit of $m_{T} \rightarrow \infty$ no mass term for these $T$ baryons is present in the lower order Lagrangian. This means that the only massive particles running in the loop are the light mesons and the result of the integral is convergent and proportional to their mass.

Using table ${ }_{1}^{1}$ one can derive the following linearly independent relations for the magnetic moments of spin $1 / 2$ baryons containing a $c$-quark:

$$
\begin{aligned}
\mu\left(\Sigma_{c}^{++}\right)+\mu\left(\Sigma_{c}^{0}\right) & =2 \mu\left(\Sigma_{c}^{+}\right) \\
\mu\left(\Sigma_{c}^{++}\right)+\mu\left(\Omega_{c}^{0}\right) & =2 \mu\left(\Xi_{c}^{+{ }^{\prime}}\right) \\
\mu\left(\Sigma_{c}^{++}\right)+2 \mu\left(\Xi_{c}^{0^{\prime}}\right) & =\mu\left(\Sigma_{c}^{0}\right)+2 \mu\left(\Xi_{c}^{+^{\prime}}\right) \\
\mu\left(\Sigma_{c}^{0}\right)+2 \mu\left(\Xi_{c}^{+^{\prime}}\right) & =\frac{1}{6 m_{c}} .
\end{aligned}
$$

Including the spin $3 / 2$ baryons one can derive six more independent relations,

$$
\begin{aligned}
\mu\left(\Sigma_{c}^{++*}\right)+\mu\left(\Sigma_{c}^{0 *}\right) & =2 \mu\left(\Sigma_{c}^{+*}\right) \\
\mu\left(\Sigma_{c}^{++*}\right)+\mu\left(\Omega_{c}^{0 *}\right) & =2 \mu\left(\Xi_{c}^{+^{\prime} *}\right) \\
\mu\left(\Sigma_{c}^{++*}\right)+2 \mu\left(\Xi_{c}^{0^{\prime} *}\right) & =\mu\left(\Sigma_{c}^{0 *}\right)+2 \mu\left(\Xi_{c}^{+^{\prime} *}\right) \\
\mu\left(\Sigma_{c}^{0 *}\right)+2 \mu\left(\Xi_{c}^{0^{\prime} *}\right) & =3\left(\mu\left(\Sigma_{c}^{0}\right)-2 \mu\left(\Xi_{c}^{+^{\prime}}\right)\right) \\
\frac{2}{3} \mu\left(\Sigma_{c}^{++*}\right)-\mu\left(\Sigma_{c}^{0}\right) & =2 \mu\left(\Xi_{c}^{+^{\prime}}\right)-\mu\left(\Sigma_{c}^{++}\right) \\
6 \mu\left(\Sigma_{c}^{+}\right)-4 \mu\left(\Sigma_{c}^{++}\right) & =-4 \mu\left(\Sigma_{c}^{+*}\right)+\frac{8}{3} \mu\left(\Sigma_{c}^{++*}\right) .
\end{aligned}
$$

The last three equations connect observables corresponding to spin $1 / 2$ and spin $3 / 2$ baryons.

Moreover, it is easy to deduce 10 analogous equations that relate baryons having a $b$-quark

$$
\begin{aligned}
& \mu\left(\Sigma_{b}^{+}\right)+\mu\left(\Sigma_{b}^{-}\right)=2 \mu\left(\Sigma_{b}^{0}\right) \\
& \mu\left(\Sigma_{b}^{+}\right)+\mu\left(\Omega_{b}^{-}\right)=2 \mu\left(\Xi_{b}^{0^{\prime}}\right)
\end{aligned}
$$

$$
\begin{aligned}
\mu\left(\Sigma_{b}^{+}\right)+2 \mu\left(\Xi_{b}^{-^{\prime}}\right) & =\mu\left(\Sigma_{b}^{-}\right)+2 \mu\left(\Xi_{b}^{0^{\prime}}\right) \\
\mu\left(\Sigma_{b}^{-}\right)+2 \mu\left(\Xi_{b}^{0^{\prime}}\right) & =-\frac{1}{12 m_{b}} \\
\mu\left(\Sigma_{b}^{+*}\right)+\mu\left(\Sigma_{b}^{-*}\right) & =2 \mu\left(\Sigma_{b}^{0 *}\right) \\
\mu\left(\Sigma_{b}^{+*}\right)+\mu\left(\Omega_{b}^{-*}\right) & =2 \mu\left(\Xi_{b}^{0^{\prime} *}\right) \\
\mu\left(\Sigma_{b}^{+*}\right)+2 \mu\left(\Xi_{b}^{-^{\prime}}\right) & =\mu\left(\Sigma_{b}^{-*}\right)+2 \mu\left(\Xi_{b}^{0^{\prime} *}\right) \\
\mu\left(\Sigma_{b}^{-*}\right)+2 \mu\left(\Xi_{b}^{-^{*} *}\right) & =3\left(\mu\left(\Sigma_{b}^{-}\right)+2 \mu\left(\Xi_{b}^{0^{\prime}}\right)\right) \\
\frac{2}{3} \mu\left(\Sigma_{b}^{+*}\right)-\mu\left(\Sigma_{b}^{-}\right) & =2 \mu\left(\Xi_{b}^{0^{\prime}}\right)-\mu\left(\Sigma_{b}^{+}\right) \\
6 \mu\left(\Sigma_{b}^{0}\right)-4 \mu\left(\Sigma_{b}^{+}\right) & =-4 \mu\left(\Sigma_{b}^{0 *}\right)+\frac{8}{3} \mu\left(\Sigma_{b}^{+*}\right),
\end{aligned}
$$

and two independent equations that relate $b$ - and $c$-baryons

$$
\begin{aligned}
\mu\left(\Sigma_{b}^{0}\right)-\mu\left(\Sigma_{b}^{+}\right) & =\mu\left(\Sigma_{c}^{+}\right)-\mu\left(\Sigma_{c}^{++}\right) \\
\mu\left(\Sigma_{c}^{++}\right)-\frac{1}{3} \mu\left(\Sigma_{c}^{++*}\right) & =\mu\left(\Sigma_{b}^{+}\right)-\frac{1}{3} \mu\left(\Sigma_{b}^{+*}\right) .
\end{aligned}
$$

From table $\overline{1}_{1}^{1}$ we see that the order $\mathcal{O}\left(1 / \Lambda_{\chi}\right)$ and $\mathcal{O}\left(1 / \Lambda_{\chi}^{2}\right)$ contributions cancel in the sum of all baryon MM within the sextet. Therefore, the average over the baryon moments measures the MM of the heavy quark, $\left\langle\mu\left(S_{Q}\right)\right\rangle=\left\langle\mu\left(S_{Q}^{*}\right)\right\rangle / 3=$ $Q_{Q} / 12 m_{Q}$.

If one has got a numerical estimate of the couplings $g_{2}$ and $g_{3}$, it is possible to derive a scale independent relation between any couple of baryons. The combination

$$
\mu\left(B_{1}\right)-\frac{\mu_{\chi}\left(B_{1}\right)}{\mu_{\chi}\left(B_{2}\right)} \mu\left(B_{2}\right)
$$

is independent of the unknown coupling $c_{S}(\mu)$ an can then be predicted. For instance

$$
\begin{aligned}
& \mu\left(\Sigma_{b}^{+}\right)+2 \mu\left(\Sigma_{b}^{-}\right)=\frac{1}{24} \frac{g_{3}^{2}}{4 \pi f_{\pi}^{2}}\left(m_{K}-m_{\pi}\right)- \\
& \frac{\Delta_{S T}}{24} \frac{g_{2}^{2}}{\left(4 \pi f_{\pi}\right)^{2}}\left(I_{K}-I_{\pi}\right)-\frac{1}{12 m_{b}} .
\end{aligned}
$$

The couplings $g_{2}$ and $g_{3}$ have been calculated theoretically. In table these computations.

There exists an experimental measurement of $g_{3}$ from CLEO coming from the decay $\Sigma_{c}^{*} \rightarrow$

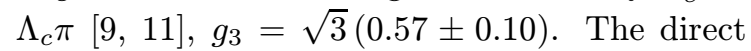
measurement of $g_{2}$ is not possible at present. 
However, the quark model relates its value to $g_{3}\left[\overline{1}_{1}^{1} \overline{1}\right.$, yielding $g_{2}=1.40 \pm 0.25$.

In order to get a numerical estimate of the left-hand side of equation $\overline{2} . \overline{1} \overline{0}_{1}^{\prime}$ we set $g_{2}=1.5 \pm$ 0.3 and $g_{3}=0.99 \pm 0.17$ and the rest of the constants as in table $\overline{4}$. We find for our best estimate of equationi $2 . \overline{10}$

$$
\mu\left(\Sigma_{b}^{+}\right)+2 \mu\left(\Sigma_{b}^{-}\right)=0.23 \pm 0.09 \mathrm{GeV}^{-1} .
$$

\section{Results for $T$-baryons $\left(s_{l}=0\right)$}

As the light quarks of the $T$-baryons are in a $s_{l}=0$ configuration, the contributions to the magnetic moments of these hadrons are $1 / m_{Q}$ suppressed [inin]. The leading term is of the form $\mu_{H Q E} / m_{Q}$ and the first chiral corrections are of order $\mathcal{O}\left(1 /\left(m_{Q} \Lambda_{\chi}\right)\right)$ and come from [i]

$$
\mathcal{L}_{(\text {long })}^{\prime}=\frac{c_{T}}{4 m_{Q}} \frac{e}{\Lambda_{\chi}} \bar{T}^{i} \sigma_{\mu \nu} Q_{i j} T^{j} F^{\mu \nu} .
$$

The contributions of order $\mathcal{O}\left(1 /\left(m_{Q} \Lambda_{\chi}^{2}\right)\right)$ have different origin:

1. there is a divergent contribution [1 14, coming through the chiral loops shown in fig. 2, which is proportional to the explicit mass splitting, $\Delta M_{Q}=3 \frac{\lambda_{2 S}}{m_{Q}}$, for the spin $1 / 2$ and spin $3 / 2$ parts of $S$-baryons [1 1 -

2. besides, one can consider a spin-symmetry breaking operator of $O\left(1 / m_{Q}\right)$;

$$
\begin{aligned}
\mathcal{L}^{\prime}= & \frac{g^{\prime}}{m_{Q}}\left[\epsilon_{i j k} \bar{T}^{i} \sigma^{\mu \nu}\left(\xi_{\mu}\right)_{l}^{j} S_{\nu}^{k l}+\right. \\
& \left.\epsilon^{i j k} \bar{S}_{k l}^{\mu} \sigma_{\mu \nu}\left(\xi^{\nu}\right)_{j}^{l} T_{i}\right],
\end{aligned}
$$

which gives rise to divergent loop diagrams, as the one in fig. 2, where one of the vertices is proportional to $g^{\prime}$.

3. further, there are finite contributions of the same order coming from the $S U(3)$-breaking operators

$$
\begin{aligned}
& e \frac{\omega_{1}}{4 m_{Q} \Lambda_{\chi}^{2}} \bar{T}^{i} \sigma_{\mu \nu} Q_{i l} \chi_{j}^{l} T^{j} F^{\mu \nu}+ \\
& e \frac{\omega_{2}}{4 m_{Q} \Lambda_{\chi}^{2}} Q_{Q} \bar{T}^{i} \sigma_{\mu \nu} \chi_{i j} T^{j} F^{\mu \nu}
\end{aligned}
$$

where, in the limit of exact isospin symme$\operatorname{try} \chi=\operatorname{Diag}\left(m_{\pi}^{2}, m_{\pi}^{2}, 2 m_{K}^{2}-m_{\pi}^{2}\right)$.
As in the case of the $S$-baryons, when all Goldstone boson loops are included, the scale $\mu$ dependence of the result of fig. 2 is canceled by the corresponding dependence of an effective $c_{T}(\mu)$. The interaction term of equation $\overline{3} . \overline{2}$ and the finite terms of equation 3.3 were first taken into account in ref. [5-5].

Similarly to what we have done in the previous paragraph we write the magnetic moment of $T$-baryons as

$$
\begin{aligned}
& \mu(B)=\frac{1}{24 m_{Q}}\left(-6 Q_{Q} \mu_{H Q E}(B)-\frac{c_{T}}{\Lambda_{\chi}} \mu_{T}(B)+\right. \\
& g_{3}^{2} \frac{3 \lambda_{2 S}}{\left(4 \pi f_{\pi}\right)^{2}} \mu_{g_{3}}(B)+6 g_{3} g^{\prime} \frac{\Delta_{S T}}{\left(4 \pi f_{\pi}\right)^{2}} \mu_{g^{\prime}}(B)+ \\
& \left.2 \frac{\omega_{1} m_{K}^{2}}{\Lambda_{\chi}^{2}} \mu_{\chi_{1}}(B)-6 Q_{Q} \frac{\omega_{2} m_{K}^{2}}{\Lambda_{\chi}^{2}} \mu_{\chi_{2}}(B)\right) .
\end{aligned}
$$

The values of the $\mu_{i}$ are written in table

$$
J_{i}=\frac{\partial}{\partial \Delta_{S T}}\left(\Delta_{S T} I\left(\Delta_{S T}, m_{i}\right)\right) .
$$

Corrections to our results for $T$-baryons are of order $\mathcal{O}\left(1 / m_{Q}^{2}\right)$ and hence negligible.

By eliminating the unknown coupling constants, one can deduce two independent relations among the magnetic moments of both T-multiplets

$$
\begin{aligned}
& m_{b} \mu\left(\Xi_{b}^{-}\right)-m_{c} \mu\left(\Xi_{c}^{0}\right)=m_{b} \mu\left(\Xi_{b}^{0}\right)-m_{c} \mu\left(\Xi_{c}^{+}\right) \\
& m_{b} \mu\left(\Lambda_{b}^{0}\right)-m_{c} \mu\left(\Lambda_{c}^{+}\right)-\frac{1}{4}=\left(2 \frac{m_{K}^{2}}{m_{\pi}^{2}}-1\right) \times \\
& {\left[m_{b} \mu\left(\Xi_{b}^{-}\right)-m_{c} \mu\left(\Xi_{c}^{0}\right)-\frac{1}{4}\right] .}
\end{aligned}
$$

In the absence of the $S U(3)$-breaking operators in equation 3.3 , the average baryon MM over the $T$ multiplet would be equal to the heavy quark MM 14.4. The result is however corrected by contributions proportional to the unknown couplings $\omega_{1}$ and $\omega_{2}{ }^{1}$ :

$$
\begin{aligned}
& \left\langle\mu\left(T_{Q}\right)\right\rangle=\frac{-1}{4 m_{c}}\left[Q_{Q}+\frac{2 \omega_{1} m_{K}^{2}}{9 \Lambda_{\chi}^{2}}\left(1-\frac{m_{\pi}^{2}}{m_{K}^{2}}\right)-\right. \\
& \left.Q_{Q} \frac{\omega_{2} m_{K}^{2}}{3 \Lambda_{\chi}^{2}}\left(2+\frac{m_{\pi}^{2}}{m_{K}^{2}}\right)\right] .
\end{aligned}
$$

\footnotetext{
${ }^{1}$ Notice that our definition of MM differs from the one in ref. $[14]$ by a factor $-1 / 4$.
} 


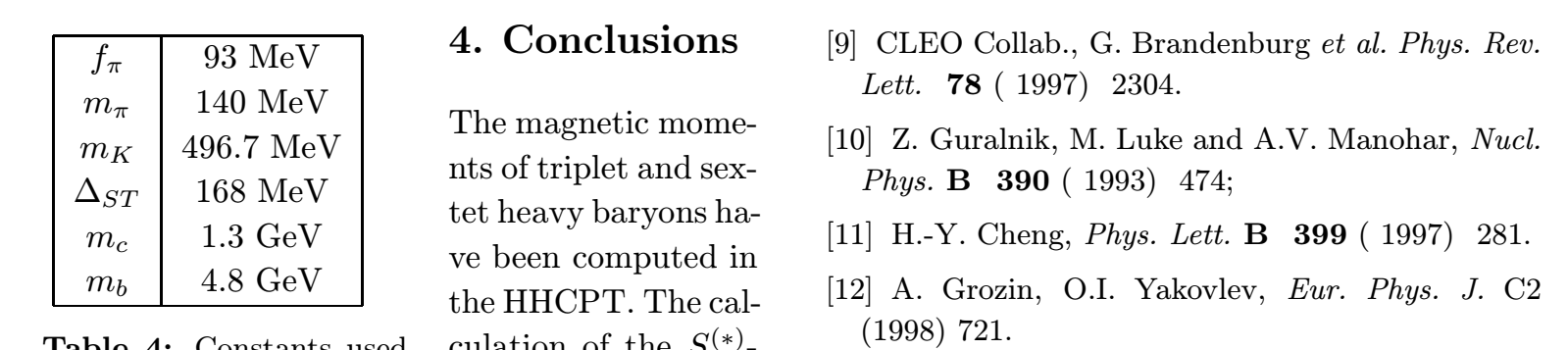

Table 4: Constants used culation of the $S^{(*)}$ in numerical estimates. _ _ baryons MM_ at the _ _ [13] S.-L. Zhu, Y.-B. Dai, iPhys. Lett. B

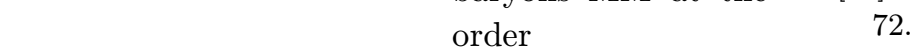

$\mathcal{O}\left(1 / \Lambda_{\chi}^{2}\right)$ involves only one new arbitrary constant, $c_{S}$. Thus it is possible to derive relations among the MM of the hadrons in the same sextet where all masses and effective couplings are eliminated. Due to heavy quark symmetry the MM of the $S$ and $S^{*}$ sextets are also related. Moreover, as $c$ and $b$ baryons are described by the same arbitrary constants, we can connect the MM of the two kinds of hadrons. The average over one sextet equals the corresponding heavy quark MM.

In the case of $T$-baryons the first corrections appear at order $\mathcal{O}\left(1 /\left(m_{Q} \Lambda_{\chi}^{2}\right)\right)$ and four arbitrary constants are required. Then we are left with only two independent relations which combine $c$ and $b$ triplets and contain $m_{c}$ and $m_{b}$. The average over one triplet equals the heavy quark MM only in the absence of $S U(3)$-breaking operators.

The measure of the magnetic moments of heavy baryons represents an experimental challenge. Nevertheless several groups are contemplating the possibility of performing it in the near future (BTeV, SELEX) [1] $\left.{ }_{1}^{1}\right]$.

\section{References}

[1] M. Wise, Phys. Rev. D45 (1992) R2188.

[2] G. Burdman and J. Donoghue, Phys. Lett. B' - 280 ( 1992$) 287$.

[3] T.M. Yan et al., 'Phys. Rev. D46 (1992) 1148' Erratum Phys. Rev. D55 ( 1997) 5851

[4] P. Cho, I'Phys. Lett. B $\mathbf{2 8 5}(\mathbf{1 9 9 2 )} 145$.

[5] M.C. Bañuls et al. hep-ph/9805488, to be published in Phys. Rev. D.

[6] P. Cho and H. Georgi, 'Phys. Lett. B $\mathbf{2 9 6}(1992)$, 1- 408; Phys. Lett. B 300 (1993) 410(E).

[7] A. Falk, 는. Phys. B $\mathbf{3} 7 \mathbf{8}(1992)$

[8] H.-Y. Cheng. et al., 'Phys. Rev. D49 (1994)!' $-2490$

[14] M.J. Savage, 'Phys. Lett. B $\mathbf{3 2 6}(1994)$ 303'.

[15] See e.g. P. Cho, 'Nucl. Phys. B $\mathbf{3 9 6}(1993) 183$ Erratum-ibid Nucl. Phys. B $\mathbf{4} \mathbf{2 1}(\mathbf{1} 994)$ 683; E. Jenkins, Phys. Rev. D54 (1996) 4515.

[16] L. Moroni and J. Russ private communications; BTeV collaboration hep-ph/9809557. 


\begin{tabular}{|c|c|c|}
\hline Baryon & $\mu_{\chi}$ & $\mu_{g_{3}}$ \\
\hline$S^{11}$ & 2 & $1+m_{\pi} / m_{K}$ \\
$\sqrt{2} S^{12}$ & $1 / 2$ & $1 / 2$ \\
$S^{22}$ & -1 & $-m_{\pi} / m_{K}$ \\
$\sqrt{2} S^{23}$ & -1 & $-\left(1+m_{\pi} / m_{K}\right) / 2$ \\
$\sqrt{2} S^{13}$ & $1 / 2$ & $m_{\pi} /\left(2 m_{K}\right)$ \\
$S^{33}$ & -1 & -1 \\
\hline Baryon & \multicolumn{2}{c|}{$\mu_{g_{2}}$} \\
\hline$S^{11}$ & & $I_{\pi}+I_{K}$ \\
$\sqrt{2} S^{12}$ & & $-I_{\pi} / 2$ \\
$S^{22}$ & & $-\left(I_{\pi}+I_{K}\right) / 2$ \\
$\sqrt{2} S^{23}$ & & $I_{\pi} / 2$ \\
$\sqrt{2} S^{13}$ & \multicolumn{3}{|c}{$I_{K}$} \\
$S^{33}$ & \multicolumn{2}{c}{} \\
\hline
\end{tabular}

Table 1: Contributions to magnetic moments of spin $1 / 2 \mathrm{c}$ and b-baryons $\left(s_{l}=1\right)$.

\begin{tabular}{|c|c|c|}
\hline Ref. & $g_{2}$ & $g_{3}$ \\
\hline$\left[10^{\prime}\right]$ & 1.88 & 1.53 \\
\hline [3) & 1.5 & 1.06 \\
\hline$[12$ & $0.83 \pm 0.23$ & $0.67 \pm 0.18$ \\
\hline$[13$ & $1.56 \pm 0.3 \pm 0.3$ & $0.94 \pm 0.06 \pm 0.2$ \\
\hline
\end{tabular}

Table 2: Theoretical estimates of $g_{2}$ and $g_{3}$.

\begin{tabular}{|c|c|c|c|}
\hline Baryon & $\mu_{T}$ & $\mu_{g_{3}}$ & $\mu_{g^{\prime}}$ \\
\hline$T_{1}$ & 4 & $J_{\pi}+J_{K}$ & $I_{\pi}+I_{K}$ \\
$-T_{2}$ & -2 & $-J_{\pi}$ & $-I_{\pi}$ \\
$T_{3}$ & -2 & $-J_{K}$ & $-I_{K}$ \\
\hline Baryon & \multicolumn{2}{|c|}{$\mu_{\chi_{1}}$} & $\mu_{\chi_{2}}$ \\
\hline$T_{1}$ & $-2 m_{\pi}^{2} / m_{K}^{2}$ & $m_{\pi}^{2} / m_{K}^{2}$ \\
$-T_{2}$ & $m_{\pi}^{2} / m_{K}^{2}$ & $m_{\pi}^{2} / m_{K}^{2}$ \\
$T_{3}$ & $2-m_{\pi}^{2} / m_{K}^{2}$ & $2-m_{\pi}^{2} / m_{K}^{2}$ \\
\hline
\end{tabular}

Table 3: Contributions to magnetic moments of spin $1 / 2 T$-baryons $\left(s_{l}=0\right)$. 


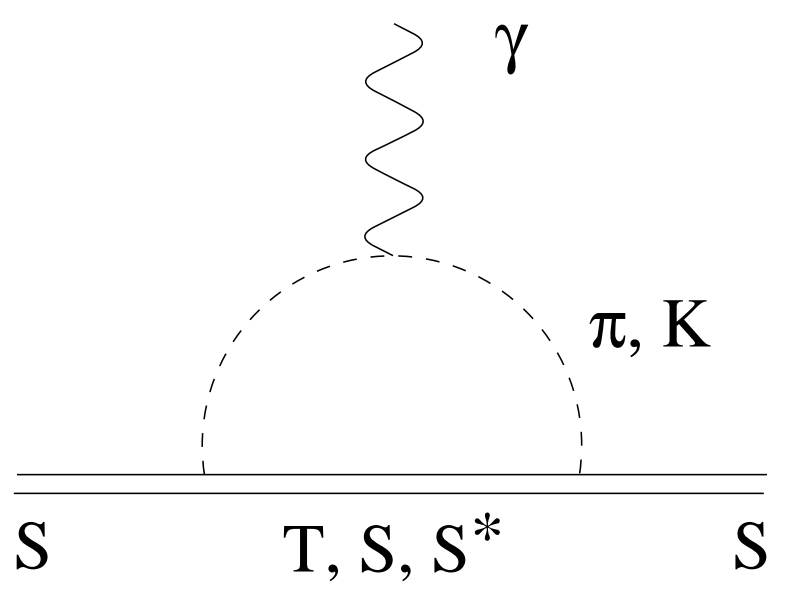

Figure 1: Meson loops contributing to $S$-baryons MM.

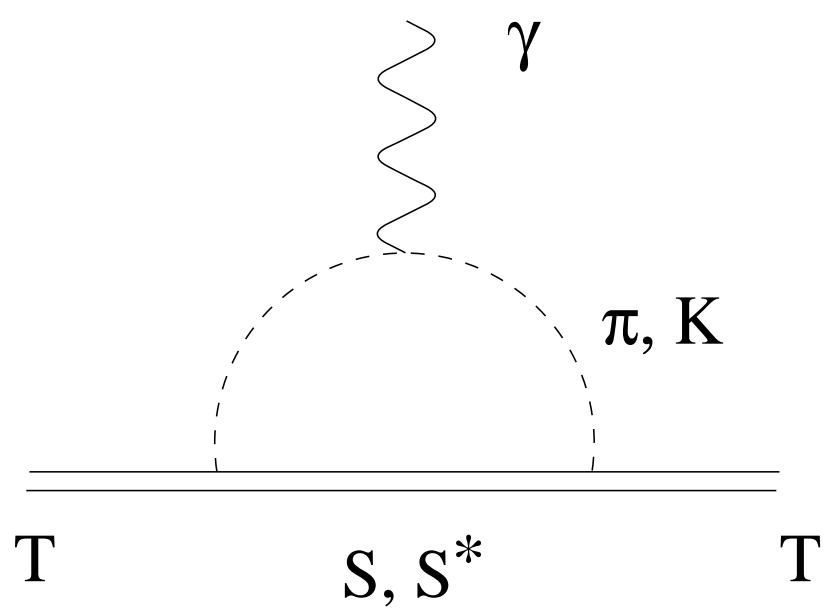

Figure 2: Meson loops contributing to T-baryons MM. 